\title{
APORTACIONES AL CONOCIMIENTO DE LA PREHISTORIA MADRILEÑA: DATACIONES POR TERMOLUMINISCENCIA DE ALGUNOS MATERIALES CERÁMICOS NEOLÍTICOS DE LA COLECCIÓN BENTO DEPOSITADOS EN EL MUSEO ARQUEOLÓGICO DE CATALUÑ ${ }^{1}$
}

\author{
CONTRIBUTION TO THE PREHISTORY OF MADRID. TERMOLUMINISCENCE \\ DATING OF SOME NEOLITHIC CERAMIC MATERIALS OF THE BENTO \\ COLLECTION KEPT IN THE ARCHAEOLOGICAL MUSEUM OF CATALONIA
}

\author{
por
}

ISABEL RUBIO DE MIGUEL

\begin{abstract}
RESUMEN La Colección Bento de prehistoria madrileña es seguramente la más importante en cuanto a cantidad de materiales, pero también una de las más completas, por lo que respecta a su composición. Entre las piezas que la conforman hay fragmentos cerámicos neolíticos con las decoraciones características (impresiones, incisiones, acanaladuras, cordones decorados) del Neolítico más antiguo hasta ahora identificado en la Meseta. Hasta el momento, el de Madrid se había fechado por comparación con éste, clasificando sus materiales (cerámica esencialmente) mediante criterios tipológicos. Ahora se dan a conocer tres dataciones de TL que se sitúan en la segunda mitad del VII ${ }^{\circ}$ milenio y en el primer tercio del $\mathrm{VI}^{\circ}$ ambos B.P. y que son las primeras fechas absolutas obtenidas para el Neolítico madrileño. Dichas fechas vienen a complementar las otras dataciones absolutas existentes para yacimientos meseteños.
\end{abstract}

\begin{abstract}
Bento Collection of madrilenian prehistory is probably the most important one, not only because of the high number of its materials, but also because it is one of the most complete in its composition. Among the pieces which form it, there are neolithic sherds with the characteristic decorations (impressions, incisions, grooves, decorated cordons) of the most ancient Neolithic identified in the Meseta. Up till now, the madrilenian Neolithic has been dated comparing with this, clasifiying its materials (mainly pottery) by typological criteria. Now we present three TL dates situated in the second half of the VII millenium and the first third of the VI both B.P., which are the first absolute chronologies obtained for madrilenian Neolithic. These complement other absolute dates existing for Meseta settlements.
\end{abstract}

\footnotetext{
1. Las fechas que damos a conocer en este artículo han sido obtenidas en el Laboratorio de Datación y Radioquímica de la U.A.M., dentro del Proyecto de Investigación : Recuperación del patrimonio arqueológico madrileño: reinterpretación de la Prehistoria reciente en la región de Madrid,06/0180/2000, de la Comunidad de Madrid y del Proyecto BHA 2001-0715 del MCYT.
} 
Palabras claves Dataciones, termoluminiscencia, Neolítico, Meseta.

Key words Dates, thermoluminiscence, Neolithic, Meseta.

La colección recopilada en los años treinta del pasado siglo (a partir de 1931, concretamente) por D. José Bento López es, sin duda, una de las más completas y voluminosas que se han reunido sobre la Prehistoria madrileña. Sus materiales (piezas líticas, cerámicas, muy pocas metálicas y fauna pleistocénica), proceden fundamentalmente de yacimientos situados en la ribera derecha del río Manzanares (distritos de Arganzuela, Mediodía y Villaverde), en una zona de particular riqueza arqueológica. ${ }^{2}$ En total, son veinticinco los areneros de los que proceden las piezas.

A pesar de la problemática que lleva aparejada cualquier colección no procedente de excavaciones sistemáticas (ausencia de referente estratigráfico, selección de las piezas, inexistencia de contexto...), J. Pérez de Barradas (1933-1935: 3) resaltaba en su momento que el Sr. Bento había anotado la procedencia de los materiales, no sólo por lo que se refería al yacimiento, sino también con respecto a las capas del terreno. Del mismo modo, destacaba la importancia de la colección y recordaba igualmente que él mismo había realizado recolecciones a lo largo de un tiempo prolongado en los yacimientos visitados por Bento, lo que avalaba el conocimiento de las particularidades de cada lugar arqueológico.

La colección fue publicada por J. Pérez de Barradas ya en los mismos años treinta (Pérez de Barradas 1933-1935), cuando ingresó en calidad de depósito en el Museo del Ayuntamiento de Madrid en 1934. Dos años después, sin embargo, D. José Bento solicitaba su devolución (Alaminos 1997: nota 38), vendiéndola a continuación al Museo Arqueológico de Cataluña con la mediación del citado Pérez de Barradas, según consta en la documentación existente en esta institución. En cualquier caso, hay documentos que atestiguan nuevamente la entrada de materiales de la referida colección en el Museo del Ayuntamiento de Madrid en 1942, así como de una defensa de Elephas de la misma procedencia en 1951.

La Colección Bento está compuesta, en su mayor parte, por materiales líticos de cronología paleolítica y muy escasos postpaleolíticos y, en menor medida, por piezas cerámicas entre las que figura un pequeño lote de recipientes completos, cerámicas que según los esquemas actuales habría que clasificar desde el Neolítico hasta el Horizonte Cogotas I. Asimismo, se tiene noticia de, al menos, cuatro piezas metálicas: una espada procedente del Arenero de La Perla y tres objetos del Arenero de Martínez (un punzón de sección rectangular, un fragmento de vaso y un cuchillo curvo)(Pérez de Barradas 1933-1935: 52). A ello habría que añadir la fauna ya citada.

En este artículo, nos proponemos dar a conocer tres dataciones de TL, las primeras fechas absolutas obtenidas para el Neolítico madrileño, a partir de fragmentos cerámicos de la colección correspondientes a dicho horizonte cultural, valorándolas en el contexto de las cronologías atribuidas al propio Neolítico madrileño por otros procedimientos y en el de las existentes para el ámbito más general de la Meseta. Podría objetarse a propósito de su procedencia que se trata de materiales antiguos no obtenidos "para contestar preguntas". Sin embargo, cabe oponer a este argumento que la importancia de la colección, la escasez de datos existente sobre el Neolítico madrileño, así como la ausencia de referentes estratigráficos en cualquier caso, hacen aconsejable no desperdiciar los datos que poseemos. Pero asimismo, el deseo de superar el ámbito de la clasificación puramente tipológica nos ha llevado a la búsqueda de dataciones absolutas para dichas cerámicas no procedentes de excavación, con todos los inconvenientes que esto pueda suponer.

2. Esta densidad de yacimientos puede comprobarse en los distintos capítulos de la obra: RUANO, E. (Dir. y Coord.) (1999-2000): "La Arqueología Madrileña en el final del Siglo XX: desde la Prehistoria hasta el año 2000", Boletín de la Asociación Española de Amigos de la Arqueologia: 39-40. 


\section{LAS CERÁMICAS NEOLÍTICAS DE LA COLECCIÓN BENTO DATADAS POR TL}

Dos (tres en cierto modo) son los yacimientos en los que se ha documentado cerámica neolítica, además de otras posteriores, y de cada uno de ellos se ha tomado una muestra para termoluminiscencia, único procedimiento de datación viable en este caso:

\section{1.- Arenero de los Vascos (Villaverde):}

- 37725 (5009): cuatro fragmentos decorados y dos lisos pertenecientes a un mismo recipiente, con decoración de líneas inciso-acanaladas y trazos oblicuos y aguada a la almagra. Miden 1: $16 \mathrm{~cm}$ de altura x $7^{\prime} 7 \mathrm{~cm}$ de ancho; $2: 5^{\prime} 5 \mathrm{~cm}$ de altura $x 4^{\prime} 5 \mathrm{~cm}$ de ancho; $3: 6^{\prime} 5 \mathrm{~cm}$ de altura x 5' $5 \mathrm{~cm}$ de ancho; 4: 4' $5 \mathrm{~cm}$ de altura x 5'5 cm de ancho; 5 (zona lisa): $5 \mathrm{~cm}$ de altura $x 4^{\prime} 5 \mathrm{~cm}$ de ancho y 6 (zona lisa): $8 \mathrm{~cm}$ de altura $x$ $5 \mathrm{~cm}$ de ancho, del que procede la muestra para datación por TL (Figura 1, a). La fecha obtenida en este caso ha sido del $6430 \pm 450$ B.P..

\section{2.- Arenero de Valdivia Oeste y Centro (Villaverde):}

- 37586 (2672 d): fragmento de galbo, de color pardo, con decoración incisa de triángulos rellenos de líneas oblicuas y trazos cortos que flanquean la base de éstos. Mide $10 \mathrm{~cm}$ de altura y $7 \mathrm{~cm}$ de ancho (Figura 1, b). La datación obtenida ha sido del $6327 \pm 480$ B.P..

\section{3.- Arenero de Valdivia Oeste (Villaverde):}

- 37744 (2626): dos fragmentos de recipiente tosco, de color pardo al exterior y negro al interior, con decoración de impresiones al interior. El conjunto mide $6 \mathrm{~cm}$ de altura y $12 ' 5 \mathrm{~cm}$ de ancho. Es posiblemente neolítico (Figura 1, c). La cronología obtenida para este fragmento ha sido del $5741 \pm 470$ B.P..

Las cerámicas neolíticas existentes en la Colección Bento se encuadran perfectamente en el contexto de las conocidas para otros yacimientos madrileños por lo que respecta a decoraciones y formas, en los casos en que éstas han podido ser determinadas (de "botella" y ovoide). Las decoraciones son, fundamentalmente, incisiones anchas o más bien acanaladuras en su mayor parte, combinadas con impresiones de punzón, cordones y nervios inciso-impresos, así como impresiones en labios y mamelones, además de incisiones en estos segundos. Los elementos de prensión citados son los de lengüieta, aunque también, en el caso de una vasija lisa se ha documentado un asa doble de cinta y en otro una pequeña asa de cinta con las decoraciones citadas. Todo ello, además de la presencia de alguna aguada a la almagra, lleva a incluirlas en el horizonte de cerámicas impresas que parece caracterizar el Neolítico más antiguo de la Meseta.

Independientemente, de estos materiales de la colección Bento conservados en el Museo Arqueológico de Cataluña, conocemos otros datos sobre alguno de los yacimientos de procedencia de los mismos, publicados en distintos momentos, que confirman los rasgos señalados. Este hecho podía documentarse, por ejemplo, en los materiales procedentes de antiguas recogidas realizadas por J. Pérez de Barradas en los años 20 y 30 en Los Vascos, dados a conocer una década más tarde (Pérez de Barradas 1941) y revisados posteriormente (Mercader, Cortés y García 1989b: 63). Se trataba de cerámica con decoraciones impresas, incisas y en relieve, a veces combinadas (Antona 1987: 56), con alguna forma de "botella", asas de cinta o tuneliformes 
y mamelones de lengüeta, además de incrustación de pasta blanca, en un caso. Por otra parte, L. Municio (1988: 309 y 319-320), en su síntesis sobre la Meseta, señalaba que la revisión hecha en su momento por $\mathrm{M}^{\mathrm{a}}$ I. Martínez Navarrete de algunos conjuntos madrileños detectó errores en la publicación de Pérez de Barradas (1933-1935) y permitió conocer que tres piezas de la Colección Bento atribuidas al Arenero de Los Vascos pertenecían en realidad al Arenero de Valdivia (Villaverde). Las decoraciones de las mismas eran incisiones amplias, flanqueadas por impresiones y cordoncillos impresos (Municio 1988: 320). Por nuestra parte hemos constatado también discrepancias en la atribución de piezas a algunos yacimientos. Recientemente, J. Jiménez (1998: fig. 6, 5 y 1999: fig. 2, 18) hace mención de la existencia de un fragmento de pseudo-cardial en Los Vascos, detectado entre los materiales ya publicados (Mercader, Cortés y García 1989b: fig. 16,1).

Finalmente, hay que recordar que uno de los escasos, por no decir el único supuesto enterramiento neolítico madrileño, es el de fosa del Arenero de Valdivia (Antona 1987: 55), sobradamente conocido, revisado también después (Jiménez 1998: figs. 8 y 9; 1999: 499 y 501 y 2001), que proporcionó una vasija en forma de "botella" y un brazalete de piedra de sección cuadrada similar al fragmento hallado posteriormente en La Deseada (Rivas-Vaciamadrid) (Díaz del Río y Consuegra 1999).

\section{LOS PARALELOS DE LAS CERÁMICAS NEOLÍTICAS DE LA COLECCIÓN BENTO}

Dentro de la misma Comunidad de Madrid, materiales similares fueron hallados en la ya citada Cueva del Aire (Fernández-Posse 1980) y en el vecino Covacho de La Higuera (Patones) (Barrio y Rubio, e.p.), en la zona de la Sierra madrileña, así como en el yacimiento del kilómetro 3'5 (derecha) de la carretera de San Martín de la Vega (Méndez y Gálvez 1984 y Mercader, Cortés y García 1989b: 22) y en el Arenero de Arganda (Mercader, Cortés y García 1989a), fundamentalmente. En otros casos, conocemos insuficientemente los materiales, como los hallados en el yacimiento del solar de la calle de La Cal (Las Carolinas, Villaverde), donde se documentaron cerámicas con decoración de punto y raya entre otras (Vigil-Escalera y Moreno 1996), en lo que presumiblemente pueda tratarse de un contexto similar.

En otro orden de cosas, podemos señalar que el Neolítico madrileño muestra un claro paralelismo con la Submeseta norte por lo que se refiere a asentamientos y materiales: cuevas de La Vaquera y La Nogalera en Segovia (Zamora 1976; Rubio y Blasco 1988-1989 y Estremera 1999 para la primera y Municio y Ruiz Gálvez 1986 para la segunda); yacimientos del valle de Ambrona (Soria) (Kunst y Rojo 1999 y Rojo y Kunst 1999) y otros de Palencia, Zamora o Valladolid (Iglesias, Rojo y Álvarez 1996). Pero también con hallazgos de la Submeseta sur, como los que se vienen produciendo en cuenca media del Tajo (Villa y Rojas 1996) e incluso Extremadura (González 1996 y 1999).

\section{DATACIONES Y SECUENCIAS PARA EL NEOLÍTICO DE LA MESETA}

Las dataciones absolutas obtenidas para el Neolítico de la Meseta se iniciaban con la radiocarbónica que fechaba los niveles inferiores de la Cueva de la Vaquera (Segovia) (Zamora 1976: 63-65) en el $3700 \pm 80$ a.C. Posteriormente (Arribas et alii 1988-1989), se dató por TL un fragmento decorado (Rubio y Blasco 1988-1989: Fig. 1, 154), similar a los hallados con anterioridad, procedente del nivel de base de las nuevas excavaciones llevadas a cabo por D. Juan Carlos Iglesias de la Universidad de Valladolid, que resultaba más reciente que la fecha de C14: $3032 \pm 336$ a. C.. El desfase entre las dos dataciones se atribuyó a tres posibles causas (Arribas et alii 1988-1989: 169): un desajuste entre ambos métodos que sería necesario comprobar, mediciones erróneas en alguno de los dos u ocupaciones esporádicas (o sucesivas), con suelos similares. Las nuevas excavaciones llevadas a cabo entre 1988 y 1995 (Estremera 1999) han permitido 
asimismo obtener dataciones radiocarbónicas para las tres fases documentadas en las mismas de las que nos interesan las dos primeras. La I (Neolítico más antiguo) se ha fechado en 4170 a. C. y la II (Neolítico reciente) en 3850 a. C. y 2900 a. C., siendo la III de tránsito al Calcolítico precampaniforme.

Los trabajos de prospección y excavación llevados a cabo en el Valle de Ambrona (Rojo y Kunst 1999 y Kunst y Rojo 1999 como publicaciones más recientes) han proporcionado nuevos e interesantes datos además de sorprendentes materiales y dataciones radiocarbónicas. El yacimiento de La Lámpara proporcionó tres fechas que se situaban en la segunda mitad del $\mathrm{V}^{\circ}$ milenio sin calibrar (segunda mitad del $\mathrm{VI}^{\circ} \mathrm{e}$ inicios del $\mathrm{V}^{\circ}$ calibrado), con un material cerámico con las técnicas y motivos habituales en la Meseta, destacando por su decoración y tratamiento simbólicos una vasija, hallada en una sepultura individual en fosa. Otro de los yacimientos, La Peña de la Abuela, una tumba monumental de carácter colectivo y el poblado de La Revilla del Campo correspondían a momentos más recientes (Kunst y Rojo 1999 y Rojo y Kunst 1999).

J. Jiménez (1998, Tabla 1) ha publicado la calibración de algunas fechas existentes para la Meseta. Parte de ellas aparecen en el cuadro adjunto, donde se recogen dataciones calibradas con que contamos en este momento y otras que no necesitan corrección (TL) para el horizonte de cerámicas inciso-acanaladas e impresas.

\begin{tabular}{|c|c|c|c|c|}
\hline Yacimiento & Asignación cultural & Fecha B.P. (sin calibrar) & Fecha a.C. & Fecha B.C. (calibrada) \\
\hline \multirow[t]{2}{*}{ La Velilla (h.) } & * & $5250 \pm 50$ & 3300 & $4090(0.74) 3980$ \\
\hline & & & & $4230(1.00) 3970$ \\
\hline \multirow[t]{2}{*}{ La Velilla (h.) } & * & $5200 \pm 55$ & 3250 & $4080(0.94) 3950$ \\
\hline & & & & $4160(0.87) 3930$ \\
\hline La Velilla (h.) & $*$ & $5070 \pm 175$ & 3120 & $4040(1.00) 3650$ \\
\hline La Velilla (h.) & $*$ & $5195 \pm 115$ & 3245 & $4160(0.74) 3930$ \\
\hline La Velilla (c.) & * & $6130 \pm 190$ & 4180 & $5260(1.00) 4840$ \\
\hline La Vaquera & $*$ & $5650 \pm 80$ & 3700 & $4690(1.00) 4340$ \\
\hline La Vaquera & $*$ & & & $3032 \pm 336 \mathrm{TL}$ \\
\hline Quintanadueñas & $*$ & $6760 \pm 130$ & 4810 & $5730(1.00) 5480$ \\
\hline La Lámpara & $*$ & $6390 \pm 60$ & 4440 & $5440(1.00) 5230$ \\
\hline La Lámpara & $*$ & $6144 \pm 46$ & 4194 & $5220(1.00) 4940$ \\
\hline La Lámpara & $*$ & $6055 \pm 34$ & 4105 & $5060(1.00) 4900$ \\
\hline Verdelpino (n. IV) & $* *$ & $7950 \pm 150$ & 6000 & $7030(1.00) 610$ \\
\hline Verdelpino (n. III) & * & $5120 \pm 130$ & 3170 & $4080(0.99) 3760$ \\
\hline Verdelpino (n. III) & $*$ & $5170 \pm 130$ & 3220 & $4090(0.88) 3790$ \\
\hline Verdelpino (n. II) & $*$ & $4630 \pm 130$ & 2680 & $3650(1.00) 3100$ \\
\hline
\end{tabular}

(* Inciso-acanaladas e impresas y ** lisas; (h.): hogares y (c.): cabaña)

Tabla 1.- Algunas dataciones absolutas (Cl4 calibradas y TL) existentes para el Neolítico más antiguo de la Meseta.

Conviene recordar, aunque aquí no se incluyan, la coincidencia de las dataciones del megalitismo de la Meseta con las de determinadas ocupaciones neolíticas del último tercio del $\mathrm{IV}^{\circ}$ milenio sin calibrar (finales del $\mathrm{V}^{\circ}$ e inicios del $\mathrm{IV}^{\circ}$ calibrado), hecho sobre el que ya habíamos expresado nuestra opinión (Rubio 
y Blasco 1988-1989). Sin embargo, el megalitismo de la Comunidad madrileña podría ser más tardío según los testimonios publicados recientemente (Díaz del Río 1996 y Jiménez 1998: Fig. 1), asociándose a momentos calcolíticos precampaniformes (o neolíticos finales).

Las secuencias culturales con que contamos fueron establecidas en distintos momentos sobre la base de los restos materiales y también de las escasas dataciones que se iban obteniendo para la Meseta en general, sin duda por la escasez y el carácter de los datos provenientes de los yacimientos madrileños. Así, por ejemplo, V. Antona (1986: 19-20) propuso una sistematización del Neolítico meseteño que, por lo que respecta a Madrid, afectaba a la primera (la que nos interesa aquí, sobre todo) y a la tercera de sus fases. En su opinión, podría definirse la más antigua por las cerámicas incisas (Cueva del Aire, Cueva de la Vaquera y Cueva de la Nogalera, ambas en Segovia), fechándose por la citada datación del 3700 a. C. del primero de los yacimientos segovianos. La tercera correspondería ya a momentos mucho más tardíos con introducción en un momento más avanzado de materiales del horizonte de Los Millares.

L. Municio, en su síntesis sobre la Meseta (1988: 325), señalaba que existían indicios en el Neolítico de este área indicativos de un proceso evolutivo en el que Atapuerca supondría el epílogo del Neolítico tipo La Vaquera, caracterizado por los mismos tipos cerámicos que venimos analizando, rompiendo así la imagen existente, en su opinión, de un bloque único

Más recientemente, J. Jiménez (1998) ha propuesto una evolución del Neolítico interior similar a la elaborada por J. Bernabéu para el área levantina. En un momento inicial (Mesolítico/ Neolítico IA, c. 6100 cal. B.C.), tendrían lugar los primeros contactos de grupos de cazadores-recolectores con productores o neolitizados próximos (Los Enebrales y Verdelpino). A continuación (Neolítico IB, c. 5500-4000/3500 cal. B.C.), se documentaría un horizonte de cerámicas impresas, inciso/acanaladas (Los Vascos y Los Enebrales/La Vaquera XXIII-XIX, Quintanadueñas II y la cabaña de La Velilla), coincidiendo con el proceso de neolitización bien desarrolladoen el resto de la Península. Posteriormente (Neolítico IIA, c. 4000/35002400-2100 cal. B.C.) se produciría un desarrollo local del Neolítico en tránsito a la primera metalurgia que conduce directamente el Calcolítico precampaniforme, con cerámicas lisas y ocupación de zonas altas (Loma del Lomo, El Jarralón y el núcleo de Entretérminos- El Rincón/ Verdelpino III y II y La Vaquera XVII-XV)). Finalmente, el Neolítico IIB (c. 4000 cal. B.C.) supondría la implantación del megalitismo de forma independiente de la fase anterior y poblamiento de áreas más próximas al Sistema Central y control de zonas de paso (osario B de La Velilla, Ciella, Pecina, Moreco, Mina y El Miradero).

Apoyándose también en las dataciones radiocarbónicas calibradas, M. Rojo y M. Kunst (1999) han situado el Neolítico interior inicial, en el que se integrarían las cerámicas decoradas, en el último tercio del $\mathrm{VI}^{\circ}$ milenio cal. B.C. (mediados del $\mathrm{V}^{\circ} \sin$ calibrar), según las dataciones de La Lámpara. Un Neolítico pleno o final se documentaría a principios del $\mathrm{IV}^{\circ}$ milenio cal. B.C., cuando había aparecido ya la inhumación colectiva (La Peña de la Abuela).

\section{DISCUSIÓN}

Las dataciones de TL que damos a conocer aquí sitúan las cerámicas neolíticas de Los Vascos y de Valdivia Oeste y Centro en la segunda mitad del VII ${ }^{\circ}$ milenio B.P. (comienzos de la segunda mitad del $V^{\circ}$ milenio: $4430 \pm 450$ y $4327 \pm 480$ A.C., aproximadamente). ${ }^{3}$ Estos fragmentos presentan la decoración características del Neolítico antiguo de la Meseta. El tercero (Valdivia Oeste), de atribución cultural menos clara desde el punto de vista tipológico, ha ofrecido sin embargo una cronología de finales del primer tercio del $\mathrm{VI}^{\circ}$ milenio B.P. (finales del primer tercio del $\mathrm{IV}^{\circ}$ milenio: $3741 \pm 470$ A.C., aproximadamente), lo que

3. Como se sabe, la cronología obtenida por TL no necesita corrección. Aquí hemos restado 2000 años para obtener las dataciones A.C., aunque para otros investigadores el año de referencia sería 1980 (Bowman 1990: 42). 
en principio parecería integrarlo en un Neolítico más reciente. Podemos considerar los fragmentos procedentes del Arenero de Valdivia como pertenecientes a dos yacimientos distintos, ya que se trata de un área realmente extensa en la que se han delimitado zonas distintas y materiales de diversas épocas.

Si consideramos el resto de las dataciones B.P. del Neolítico antiguo de la Meseta, éstas se distribuyen a lo largo del VII ${ }^{\circ}$ milenio, fundamentalmente en su segunda mitad (Quintanadueñas, La Lámpara, La Velilla y La Vaquera, así como Los Vascos y Valdivia Oeste y Centro), del VI ${ }^{\circ}$ (La Vaquera, La Velilla, Verdelpino y Valdivia Oeste) e, incluso, dos en la primera mitad del $V^{\circ}$ (La Vaquera y Verdelpino). En ese sentido, las que aquí presentamos encajarían perfectamente entre las ya conocidas. Dataciones de TL recientemente obtenidas para cerámicas similares procedentes de uno de los abrigos de la zona del río Duratón, que la Dra. $M^{\mathrm{a}} \mathrm{R}$. Lucas presenta en este mismo Homenaje al Dr. Pellicer, vienen a corroborar la cronología ofrecida por las nuestras.

Con motivo de la publicación de la datación por TL de la Cueva de la Vaquera (Rubio y Blasco 1988-1989), observábamos un desfase entre ésta y la radiocarbónica a.C., desfase que se mantiene con la más antigua (Fase I), de las publicadas después. Sin embargo, esta misma y la del 3850 a.C. de su Fase II, al igual que la de La Velilla del 4180 a.C. y las de La Lámpara a.C. se hallarían más en sintonía con las nuestras, siendo más bajas las restantes de La Velilla y las de Verdelpino.

Si tenemos en cuenta las dataciones calibradas de la Meseta, la banda temporal delimitada por ellas para un Neolítico antiguo (IB) con materiales similares a los nuestros se situaría entre el 5500 y el 4000/3500 cal. B.C., como establece J. Jiménez (1998), quien precisamente incluye Los Vascos en este momento. Esta amplia franja deberá matizarse a medida que dispongamos de más datos, pero por el momento incluso nuestra fecha más reciente encajaría cómodamente en ella. La necesidad de perfilar estos matices la demuestra también el que, tanto el Neolítico inicial atestiguado en Ambrona, fechado en el último tercio del $\mathrm{VI}^{\circ}$ milenio calibrado, como el pleno o final, situado a comienzos del IV ${ }^{\circ}$ milenio calibrado cuando ya había aparecido el enterramiento colectivo, se encuadran en la banda temporal determinada por J. Jiménez para el horizonte más antiguo.

En todo caso, la diferencia más acusada para las nuestras se produce con las dataciones calibradas de La Lámpara y Quintanadueñas, que también se alejan del resto de las radiocarbónicas corregidas, hallándose entonces las de Madrid más acordes con las de La Velilla, Verdelpino o incluso La Vaquera cal. B.C..

Resumiendo y como conclusión, cabría señalar que las tres dataciones que presentamos aquí son las primeras para el Neolítico madrileño, lo que las hace especialmente interesantes, aunque evidentemente no suplan más que en una mínima parte las carencias de información existentes para el mismo. Su sintonía con otras dataciones B.P. para el Neolítico de cerámicas inciso-acanaladas e impresas es evidente. Si convertimos dichas dataciones en fechas a.C., las de TL parecen hallarse también próximas a las mismas, sin embargo al calibrar las radiocarbónicas surgen las discrepancias. Parece pues que las dataciones obtenidas por TL, al menos en este caso, y en el de los yacimientos segovianos de La Vaquera y la zona del Duratón, tienden a ser más bajas que las calibradas de C14.

En todo caso, podrían plantearse varias explicaciones para esta diferencia, especialmente con las dataciones calibradas de La Lámpara. Una de ellas podría estar relacionada con los procedimientos mismos de datación, lo que habría que demostrar. Pero también cabría sugerir la existencia de diversos horizontes incluso dentro de este primer neolítico, como parece advertirse, o quizá, de cronologías diferentes para zonas diferentes de la misma Meseta. En cualquier caso, el interés de estas primeras fechas absolutas del Neolítico madrileño radica en que vienen a apoyar una cronología más elevada que la tradicionalmente otorgada al del interior de la Península. En definitiva, es posible afirmar en el momento actual que el Neolítico inicial de la Meseta, caracterizado por conjuntos de cerámicas decoradas (impresas, incisas, con aguadas a la almagra), puede ser fechado desde la mitad del $\mathrm{VI}^{\circ}$ milenio cal. B.C. (odesde el $\mathrm{V}^{\circ}$ a.C. y A.C. por TL) pordos procedimientos de cronología absoluta, restando por determinar su evolución interna y por perfilar con mayor seguridad, al mismo tiempo, la relación entre ambos tipos de datación. 


\section{BIBLIOGRAFÍA}

ALAMINOS, E. (1997): Actas del Patronato del Museo Municipal (1927-1947), Museo Municipal de Madrid. ALCOLEA, J. et alii (1992): "La Cueva de las Avispas. Un yacimiento con arte rupestre en la provincia de Madrid", Boletín de la Asociación Española de Amigos de la Arqueología 32: 19-22.

_ et alii (1994): "Las representaciones esquemáticas del Abrigo de Belén (Torremocha, Madrid)", Estudios de Prehistoria y Arqueología Madrileñas 9: 29-32.

ANTONA, V. (1986): "Aproximación a la problemática del Neolítico de la Meseta: Una propuesta de secuencia cultural", Wad-al-Hayara 13: 9-42.

_- (1987): "El Neolítico", en 130 años de arqueología madrileña. Madrid: 109-120.

ARRIBAS, J.G. et alii (1988-1989): "Datación por termoluminiscencia y análisis mineralógico de materiales arqueológicos procedentes del yacimiento Cueva de la Vaquera (Segovia)", Zephyrus XLI-XLII: 161-169.

BARRIO, J. y RUBIO, I. (e. p.): "El yacimiento neolítico del Covacho de la Higuera (Patones, Madrid). Su valoración en el contexto de la Meseta", Estudios de Prehistoria y Arqueología madrileñas.

BERNABÉU, J. (1988): El Neolítico en las comarcas meridionales del País Valenciano, LÓPEZ, P. (Coord.), El Neolítico en España: 131-166, Madrid.

— y MARTÍ, B.(1992): "El País Valenciano de la aparición del Neolítico al horizonte campaniforme", Aragón/litoral mediterráneo. Intercambios culturales durante la Prehistoria: 213-234, Zaragoza.

BOWMAN, S. (1990): Radiocarbon dating, British Museum Publications.

DELIBES, G. y ROJO, M. (1987): "Los sepulcros colectivos del Duero medio y Las Loras y su conexión con el foco dolménico riojano", en El Megalitismo en la Península Ibérica: 181-197, Madrid.

— , ALONSO, M. y GALVÁN, R. (1986): "El Miradero: un enterramiento colectivo tardoneolítico de Villanueva de los Caballeros (Valladolid)", Estudios en Homenaje al Prof. Beltrán: 227-236.

DÍAZ DEL RÍO, P. (1996): "Hábitat agrario y rendimientos diferidos: el caso de La Esgaravita (Alcalá de Henares)", Reunión de Arqueología Madrileña, 25-26 de enero: 115-118.

- y CONSUEGRA, P. (1999): "Primeras evidencias de estructuras de habitación y almacenaje neolíticas en el entorno de la Campiña madrileña: el yacimiento de "La Deseada" (Rivas-Vaciamadrid, Madrid)", II Congrés del neolític a la Península Ibérica (7-9 de abril): 251-257, Universidad de Valencia.

ESTREMERA, M ${ }^{a}$ S. (1999): "Sobre la trayectoria del Neolítico interior: precisiones a la secuencia de La Vaquera", II Congrés del neolític a la Península Ibérica (7-9 de abril): 245-250, Universidad de Valencia.

FERNÁNDEZ POSSE, Mª D. (1980): "Los materiales de la Cueva del Aire (Patones, Madrid)", Noticiario Arqueológico Hispano 10: 41-64.

GARCÍA-GELABERT, Mª.(1996): "Carta arqueológica de Alpedrete", Reunión de Arqueología Madrileña ( 25-26 de enero): $269-272$.

GONZÁLEZ, A. (1996): "Asentamientos neolíticos en la Alta Extremadura”, I Congrès del Neolític a la Península Ibérica, Gavà-Bellaterra, 1995, 2: 697-705.

_ (1999): "Comunidades neolíticas en los riberos Alto-Extremeños del Tajo", II Congrés del neolític a la Península Íbérica (7-9 de abril): 531-540, Universidad de Valencia.

IGLESIAS, J.C., ROJO, M. y ÁLVAREZ, V. (1996): "Estado de la cuestión sobre el neolítico en la Submeseta norte", I Congrés del Neolític a la Península Ibérica, (Gavá-Bellaterra, 1995) 2: 721-734.

JIMÉNEZ, J. (1998): "La neolitización en la cuenca alta del Tajo. Nuevas propuestas interpretativas para el Neolítico de la Meseta", Complutum 9: 27-47.

, 1999: "El proceso de neolitización del interior peninsular", II Congrés del neolític a la Península Ibérica (7-9 de abril): 493-501, Universidad de Valencia.

— , 2001: "El yacimiento de Valdivia (Madrid). Nuevos elementos materiales para la interpretación del Neolítico del interior peninsular", Estudios de prehistoria y arqueología madrileñas 11: 59-68. 
et alii (1997): "Nuevos datos sobre el Neolítico meseteño: la provincia de Guadalajara", II Congreso de Arqueología Peninsular (Zamora, 24-28 de septiembre de 1996) II: Neolítico, Calcolítico y Bronce: 33-47.

KUNST, M. y ROJO, M. (1999): "El Valle de Ambrona: un ejemplo de la primera colonización Neolítica de las tierras del interior peninsular", II Congrés del neolític a la Península lbérica (7-9 de abril): 259-270, Universidad de Valencia.

MÉNDEZ, A. y GÁLVEZ, P. (1984): "Nuevos materiales de la Edad del Bronce en el término de Madrid. El yacimiento del Km 3'5 izquierda de la carretera de San Martín de la Vega”, Estudios de Prehistoria y Arqueología Madrileñas: 33-73.

MERCADER, J., CORTÉS, A.F. y GARCÍA, Ma E. (1989a): "Materiales neolíticos en el valle del Jarama (Arganda, Madrid)", Trabajos de Prehistoria 46: 255-260.

,$--\mathrm{y}-(1989 \mathrm{~b})$ : "Nuevos yacimientos neolíticos y de la Edad del Bronce en el término municipal de Madrid", Estudios de Prehistoria y Arqueología Madrileñas: 21-82.

MUNICIO, L. (1988): “El Neolítico en la Meseta Central Española”, en LÓPEZ, P. (Coord.), El Neolítico en España: 299-327. Madrid.

— y RUIZ GÁLVEZ, Mª L. (1986): “Un nuevo yacimiento neolítico en la Meseta norte: las cerámicas decoradas de la cueva de La Nogalera (Villaseca, Segovia)", Numantia 2: 143-157.

PÉREZ DE BARRADAS, J. (1933-1935): "Nuevos estudios sobre Prehistoria madrileña. I.- La colección Bento", Anuario de Prehistoria Madrileña 4-6: 3-90.

— (1941): "Poblado prehistórico de Los Vascos (Villaverde, Madrid)", AMSEAEP XVI, Cuadernos I y II: $158-160$.

ROJO, M. y KUNST, M. (1999): "La Lámpara y la Peña de la Abuela. Propuesta secuencial del Neolítico Interior en el ámbito funerario", II Congrés del neolític a la Península Ibérica (7-9 de abril): 503-512, Universidad de Valencia.

RUANO, E. (Dir. y Coord.)(1999-2000): La Arqueología Madrileña en el final del Siglo XX: desde la Prehistoria hasta el Año 2000, Boletín de la Asociación Española de Amigos de la Arqueología 39-49.

RUBIO DE MIGUEL, I. (1983): "Del Paleolítico al inicio de la Edad de los Metales en Madrid", Boletín de la Asociación Española de Amigos de la Arqueología 18: 4-14.

(1985): "El Neolítico en Madrid. Estado de la cuestión", Madrid, objetivo cultural: 23-29. Madrid. (1999-2000): "Las primeras sociedades agrícolas en Madrid. Neolítico y Calcolítico precampaniforme", RUANO, E. (Dir. y Coord.): La Arqueología Madrileña en el final del Siglo XX: desde la Prehistoria hasta el Año 2000, Boletín de la Asociación Española de Amigos de la Arqueología 39-49: 105-126.

— y BLASCO, C. (1988-1989): “Análisis cerámicos de la Cueva de la Vaquera (Torreiglesias, Segovia)”, Zephyrus XLI-XLII: 149-160.

SÁNCHEZ MESEGUER, J. et alii (1983): "El Neolítico y la Edad del Bronce en la Región de Madrid", Arqueología y Paleoecología 3.

VIGIL-ESCALERA, A. y MORENO, A. (1996): "Materiales arqueológicos de la calle de la Cal, números 15/17", Reunión de Arqueología Madrileña, 25-26 de enero: 91-93.

VILLA, J.R. y ROJAS, J.M. (1996): “Aportación al conocimiento del Neolítico en la cuenca media del Tajo", I Congrès del Neolític a la Península Ibérica, Gavà-Bellaterra, 2: 707-714.

ZAMORA, A. (1976): Excavaciones en la cueva de La Vaquera, Torreiglesias, Segovia (Edad del Bronce), Segovia. 


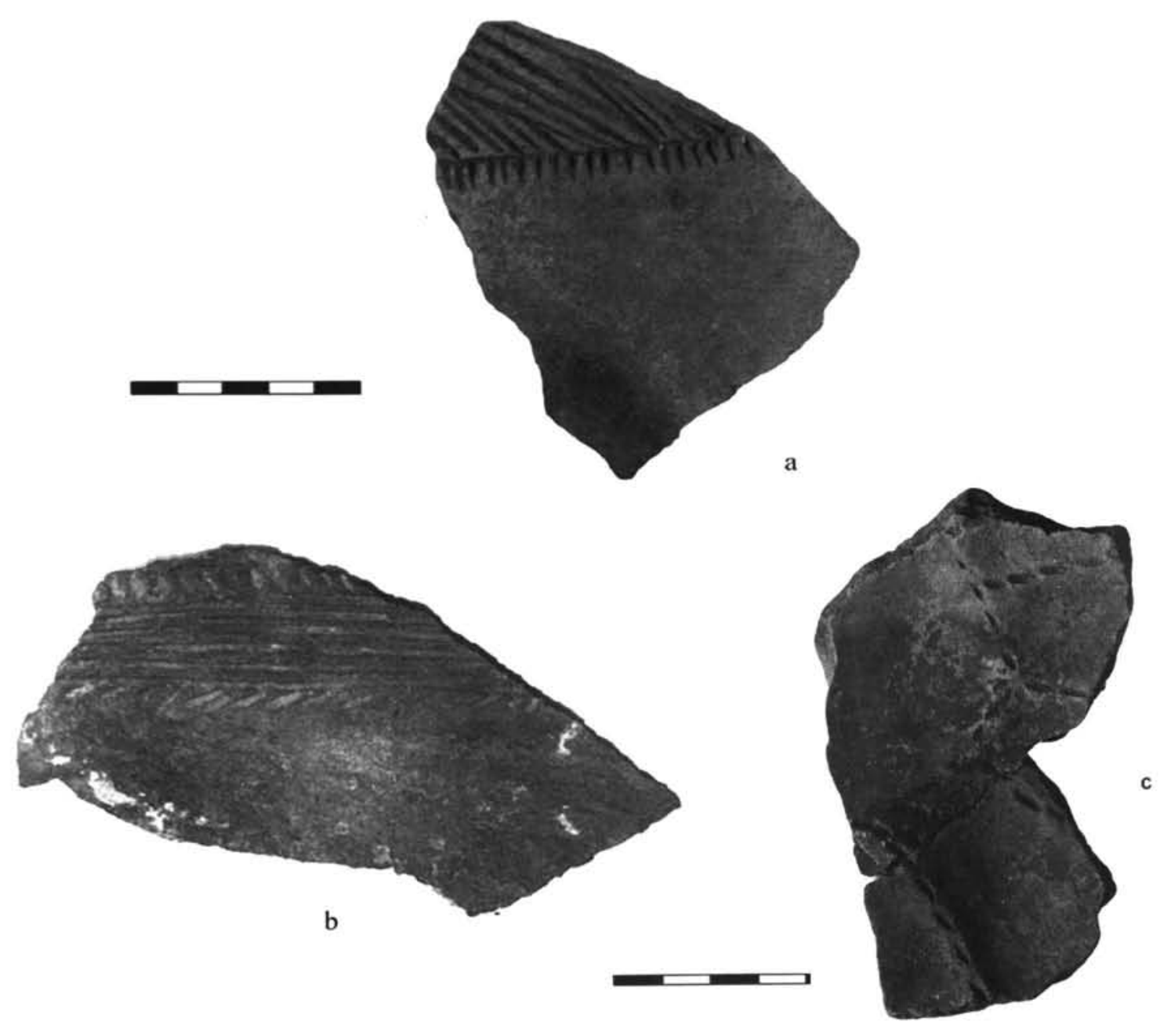

Figura 1.- Fragmentos cerámicos neolíticos de la Colección Bento de Prehistoria madrileña datados por TL (a: Arenero de Los Vascos, b: Arenero de Valdivia Oeste y Centro y c: Arenero de Valdivia Oeste). 